\title{
QUADRATIC OPTIMIZATION OF LIFE AND PENSION INSURANCE PAYMENTS
}

\author{
BY
}

\author{
Mogens STEFFEnSEN
}

\begin{abstract}
Quadratic optimization is the classical approach to optimal control of pension funds. Usually the payment stream is approximated by a diffusion process. Here we obtain semiexplicit solutions for quadratic optimization in the case where the payment process is driven by a finite state Markov chain model commonly used in life insurance mathematics. The optimal payments are affine in the surplus with state dependent coefficients. Also constraints on payments and surplus are studied.
\end{abstract}

\section{KEYWORDS}

Markov chain, Regulation of payments, Linear regulator, Bellman equations, Constraints.

\section{INTRODUCTION}

Stochastic control in life and pension insurance concentrated until recently on control of pension funds. Defined contribution schemes and defined benefit schemes leave the benefits and the contributions, respectively, as variables partly decided by the fund manager. In addition, decisions on allocation of assets may be integrated in the problem. The institutional conditions for pension funds may be rather involved. It is by no means clear how the objectives of the fund manager, the employer who pays (parts of) the premium, and the employed who receives the benefits, should be reflected in the objective of the control problem.

The usual framework of control of pension funds is the one given in probably the most studied control problem, the linear quadratic optimal control problem or the linear regulator problem. The object in this class of control problems is to control, at the same time, the position of a certain process and the force with which this process is regulated. The object function punishes quadratic deviations from some targets of the controlled process and the controlled rate of regulation, respectively. This object function is widely used partly because 
of its mathematical tractability and partly because it makes sense in certain engineering applications.

In the context of pension funding the regulated process represents some notion of surplus whereas the regulation itself represents payments. These are premiums or benefits depending on the type of scheme. Obviously, only payments which are allowed to depend on the performance of the pension fund, are open for regulation. Defined payments like e.g. guaranteed benefits do not count as decision variables. A state of the art exposition of stochastic control of pension funds is given in Cairns (2000) which is partly a survey article gathering results of several authors. The literature contains solutions to several variants of the problem. From the reference list in Cairns (2000) we draw the reader's attention to the contributions by O'Brien (1986), Dufresne (1989), and Haberman et al. (1994).

The linear regulator approach has been standard in engineering and has found its application in insurance through pension funding. However, it was not widely used as an approach to dynamic financial decision problems like e.g. consumption-investment problems. There, the most popular approach is the one taken by Merton $(1969,1971)$. This is based on optimal utility of future wealth or surplus, or, in case of introduction of consumption, utility of future consumption rates.

In Steffensen (2004), this utility optimization approach to financial decision making was applied to the problem of the life insurance company regulating surplus by adjusting regulative payments. There the set-up differs from the classical one in finance by formalizing the process of accumulated consumption as an insurance payment stream. This stream includes payment rates and lump sum payments linked to the state of an insurance policy (portfolio). Modelling the policy by a general finite state Markov chain allows for various applications in various types of insurance and on various levels of individualization of policies in the portfolio.

In the linear regulator approach to stochastic control of pension funds, the payments are usually modelled on an aggregate portfolio level by modelling the risk in payments by a diffusion term. Steffensen (2004) shows that for power utility optimization, the structure of the object function is reflected in a statedependent value function and state-dependent optimal payments. A natural question is now: Taking the linear regulator approach, will the structure of the object function again be reflected in a state-dependent value function and state-dependent optimal payments? This article answers yes to this question.

This answer is a part of the motivation for this article. A strong conclusion is that the insurance company can apply the quadratic optimization criteria for regulation of payments at any sub-portfolio level, even at the level of the individual, and maintain a simple regulation rule. This is useful if the insurance company wishes to, or is forced to, account for or manage each sub-portfolio separately. A weaker conclusion is that if the insurance company applies the linear regulation of payments based on diffusion modelling, then this regulation can partly be argued for even at a sub-portfolio level. Apart from these 
immediate applications to individualization of accounts, the article is a contribution to the general discussion on optimal design of payments.

One general drawback of quadratic approaches to investment problems is the counter-intuitive conclusion that if the surplus is above the surplus target, then one should try to loose money on the financial market. This drawback appears in quadratic hedging approaches in finance as well as in linear regulation of pension funds. One could then choose to act only when the surplus is below its target. In Steffensen (2001), this problem is resolved by punishing deviations of the deflated surplus instead of the nominal surplus. In this article, we resolve the problem simply by disregarding the asset allocation as a decision variable. Anyway, our object is to generalize the modelling of payments.

In general, linear regulator problems lose their mathematical tractability when introducing constraints on the controls or the controlled processes. Some constraints can be allowed for while some other constraints, with clear applications, make the problem much harder. An important example is to constrain the regulation of payments to be to the policy holder's benefit. This means that the fund manager or insurance company is allowed to pay out positive surplus only (by increasing benefits or decreasing premiums) and is not allowed to collect deficits. Steffensen (2001) obtains results in this direction and also shows that a terminal expectation condition is easily taken care of by a Lagrange multiplier. We approach some tractable constraints on the surplus and the payments at the end of the article.

The outline of the article is as follows. In Section 2 the dynamics of the surplus are introduced, and in Section 3 these dynamics are motivated by considering some notions of surplus introduced previously in the literature. In Section 4 the preferences are formalized in the object function. Section 5 contains the main results of the article. In Section 6 and Section 7 we show how to handle certain constraints on the payments and the surplus, respectively. At the end of Sections 3, 5, 6, and 7 we present a cross-sectional continued example which presents the machinery at work. This example also serves as motivation.

\section{The Dynamics of the Surplus}

We take as given a probability space $(\Omega, \mathcal{F}, P)$. On the probability space is defined a process $Z=(Z(t))_{0 \leq t \leq T}$ taking values in a finite set $g=\{0, \ldots, J\}$ of possible states and starting in state 0 at time 0 . We define the $J$-dimensional counting process $N=\left(N^{k}\right)_{k \in \jmath}$ by

$$
N^{k}(t)=\#\{s \mid s \in(0, t], Z(s-) \neq k, Z(s)=k\},
$$

counting the number of jumps into state $k$ until time $t$. Assume that there exist deterministic functions $\mu^{j k}(t), j, k \in \mathcal{I}$, such that $N^{k}$ admits the stochastic intensity process $\left(\mu^{Z(t) k}(t)\right)_{0 \leq t \leq T}$ for $k \in \mathcal{I}$, i.e. 


$$
M^{k}(t)=N^{k}(t)-\int_{0}^{t} \mu^{Z(s) k}(s) d s
$$

constitutes a martingale for $k \in \mathcal{J}$. Then $Z$ is a Markov process. The reader should think of $Z$ as a policy state of a life insurance contract, see Hoem (1969) for a motivation for the set-up.

Based on the probability theoretical framework above we now go directly to the dynamics of the surplus. This will allow the reader to accept the dynamics and comprehend the control problem without necessarily having it grounded in the notions of surplus studied by Norberg (1999) and Steffensen (2000). In the following section we link the surplus dynamics introduced below with the notions of surplus studied there. However, already now we need some clarification of terminology: Throughout the article, the contributions are added to the surplus. Working with e.g. the notion of surplus introduced in Section 3, these contributions stem from the realized payments compared to what is taken into account in the liability valuation. This is in contrast to the usual terminology of pension funding where the contributions are usually the premium payments. The dividends, which may in general be positive or negative, are subtracted from the surplus. The dividends regulate the payments that are taken into account in the liability valuation, and adapt these payments to the development of the policy.

We introduce the nominal surplus process $X^{\prime}$ given by

$$
\begin{aligned}
& d X^{\prime}(t)=r(t) X^{\prime}(t) d t+d C^{\prime}(t)-d D^{\prime}(t), \\
& X^{\prime}(0-)=0,
\end{aligned}
$$

where $r$ is a deterministic interest rate process and the contributions $C^{\prime}$ and the dividends $D^{\prime}$ follow the dynamics

$$
\begin{aligned}
& d C^{\prime}(t)=c^{Z(t)^{\prime}}(t) d t+\sum_{k \in \mathcal{I}} c^{Z(t-) k^{\prime}}(t) d N^{k}(t)+\sum_{n \in\{0, T\}} \Delta C^{Z(t))^{\prime}}(t) d \varepsilon^{n}(t), \\
& d D^{\prime}(t)=d^{\prime}(t) d t+\sum_{k \in \mathcal{I}} d^{k \prime}(t) d N^{k}(t)+\sum_{n \in\{0, T\}} \Delta D^{\prime}(t) d \varepsilon^{n}(t),
\end{aligned}
$$

where $\varepsilon^{n}(t)=I(t \geq n)$ indicates that $t \geq n$. Here the coefficients of the contributions $c^{j^{\prime}}(t), c^{j k^{\prime}}(t)$, and $\Delta C^{j^{\prime}} t$ are deterministic functions. The coefficient $c^{j^{\prime}}(t)$ represents the rate of contributions during sojourn in state $j$ at time $t$. The coefficient $c^{j k^{\prime}}(t)$ represents the lump sum contribution when jumping from state $j$ to state $k$ at time $t$. Finally, the coefficient $\Delta C^{j^{\prime}}(t)$ represents a lump sum contribution at the deterministic time point $t$ during sojourn in state $j$. We allow for lump sum contributions at deterministic time points only at time 0 and $T$.

In (1) the initial condition $X^{\prime}\left(0^{-}\right)=0$ defines the surplus just prior to time 0 such that the surplus at time 0 can be expressed through the dynamics of $C$ and $D$, namely, $X^{\prime}(0)=\Delta C^{\prime}(0)-\Delta D^{\prime}(0)$. The source of surplus contributions 
is a possible difference between an anterior measure and a posterior measure of a set of assumed payments. As future payments turn into past payments, the contributions are realized. Depending on the measures these contributions may be systematic and/or purely erratic. In the next section we consider a surplus definition with a concrete example of these measures.

The stochastic differential equation for the surplus (1) can be considered as a controlled stochastic differential equation with the control being the coefficients in the dividend process $D^{\prime}$. The insurance company is allowed to choose these coefficients such that there exists a solution to the stochastic differential equation (1). Then we say the dividend process $D^{\prime}$ belongs to a set $\mathcal{A}$.

We have decorated the nominal processes above with a prime to ease the notation for the corresponding discounted processes introduced below. We namely, instead of working with nominal contributions, nominal dividends, and nominal surplus, work with the discounted versions defined by

$$
\begin{aligned}
d C(t) & =e^{-\int_{0}^{t} r} d C^{\prime}(t) \\
& =c^{Z(t)}(t) d t+\sum_{k \in \mathcal{I}} c^{Z(t-) k}(t) d N^{k}(t)+\sum_{n \in\{0, T\}} \Delta C^{Z(t)}(t) d \varepsilon^{n}(t), \\
d D(t) & =e^{-\int_{0}^{t} r} d D^{\prime}(t) \\
& =\delta(t) d t+\sum_{k \in \mathcal{I}} \delta^{k}(t) d N^{k}(t)+\sum_{n \in\{0, T\}} \Delta D(t) d \varepsilon^{n}(t) . \\
X(t) & =e^{-\int_{0}^{t} r} X^{\prime}(t) .
\end{aligned}
$$

Note that the deterministic quantities $c^{j}(t)=e^{-\int_{0}^{t} r} c^{j^{\prime}}(t), c^{j k}(t)=e^{-\int_{0}^{t} r} c^{j k^{\prime}}(t)$ etc. are hereby defined. Then, given a dividend process $D^{\prime} \in \mathcal{A}$, the controlled stochastic differential equation describing the surplus is given by

$$
\begin{aligned}
& d X^{D}(t)=d C(t)-d D(t), \\
& X^{D}(0-)=0 .
\end{aligned}
$$

Note that, in contrast to the usual situation in finance where the surplus (wealth) and the dividend payments (consumption) are constrained to be positive, we impose no such constraints at this stage. This is one fundamental difference between the set-up in this article and the set-up in Steffensen (2004). There the surplus and the dividend payments were constrained to be positive such that a certain solvency constraint was fulfilled and such that dividends were to the benefit of the policy holder.

The absence of constraints on the surplus and the dividends (and the technical valuation basis) limits our results to pension funding. This is in contrast to participating life insurance where the insurance company would need a positive surplus to fulfill certain solvency requirements and where the dividends are restricted to be to the benefit of the policy holder. In practice there may 
also be constraints in pension funding. Though similar in structure to participating life insurance, they will be less strict and we choose to disregard these. See also Steffensen (2000) for a similar clear distinction between participating life insurance and pension funding.

Note that depending on the final form of $D$, the dividends may both change premiums and/or benefits. Thus, we do not specify whether we have a so-called defined contributions scheme, using this term for the situation where the premiums are fixed and dividends affect the benefits only, or a so-called defined benefits scheme, using this term for the situation where the benefits are fixed and dividends affect the premiums only. Below we see how these different cases are obtained by an according specification of the preferences.

\section{The Surplus and Life Insurance Payment Streams}

In this section we link the surplus dynamics introduced in the previous section with some notions of surplus studied in Norberg (1999) and Steffensen (2000). This is to be seen as examples of how the coefficients of the contribution process could be specified.

One part of the payment process of an insurance contract is the guaranteed payment process. Denoting by $B(t)$ the accumulated guaranteed payments to the policy holder over $[0, t]$, the guaranteed payments are described by

$$
d B(t)=b^{Z(t)}(t) d t+\sum_{k \in \mathcal{I}} b^{Z(t-) k}(t) d N^{k}(t)+\sum_{n \in\{0, T\}} \Delta B^{Z(t)}(t) d \varepsilon^{n}(t) .
$$

See Steffensen (2004) for an interpretation of the various elements of $B$, noting that there the process of guaranteed payments $B$ is denoted by $\hat{B}$.

The guaranteed payment process $B$ constitutes typically only one part of the total payment process. The insurance company adds to the guaranteed payments an additional dividend payment process depending on the performance of the insurance policy or a set of policies. The insurance company decides on this additional payment process within any legislative constraints there may be. The dividend process was introduced in the previous section. The guaranteed payments $B$ and the dividend payments $D$ constitute the total payment process experienced by the policy holder.

Since different notation has been used for guaranteed payments and dividends in the literature we present here a small notation translator:

Steffensen $(2000,2004) \quad$ Norberg (1999), here

Guaranteed payments

Dividends

Total payments
$\hat{B}$

$\tilde{B}$

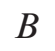

$D$

$$
B=\hat{B}+\tilde{B} \quad B+D .
$$


We introduce a notion of surplus along the lines of Steffensen (2000). There, the surplus is defined for a given deterministic valuation basis, i.e. a set of discount rate and intensity processes $\left(r^{*}, \mu^{*}\right)$, by

$$
X^{\prime}(t)=-\int_{0-}^{t} e^{\int_{s}^{t} r} d(B(s)+D(s))-V^{* Z(t)}(t),
$$

where the statewise reserves according to the valuation basis $\left(r^{*}, \mu^{*}\right)$ are given by

$$
V^{* j}(t)=E^{*}\left[\int_{t}^{T} e^{-\int_{t}^{s} r^{*}} d B(s) \mid Z(t)=j\right], j \in \mathcal{g} .
$$

Here, $E^{*}$ denotes expectation with respect to the probability measures under which $N^{k}$ admits the intensity processes $\left(\mu^{*} Z(t) k(t)\right)_{0 \leq t \leq T}$. Definition (3) corresponds to the surplus introduced in Steffensen (2000) for the case where the payments are invested in a portfolio with return rate on investment $r$. Definition (3) follows the lines of the (individual) surplus introduced in Norberg (1999) as well. However, in Norberg (1999) the dividends paid in the past are not accounted for on the asset side and the valuation basis $\left(r^{*}, \mu^{*}\right)$ is fixed to be the first order valuation basis $(\hat{r}, \hat{\mu})$ introduced below.

The insurance company lays down the guaranteed payment process $B$ on a so-called first order valuation basis $(\hat{r}, \hat{\mu})$. This means that the guaranteed payments are set to fulfill the so-called equivalence relation $V^{* 0}(0-)=0$ for $\left(r^{*}, \mu^{*}\right)=(\hat{r}, \hat{\mu})$. In participating life insurance one would usually impose a constraint on the first order basis such that the first order reserves $V^{* j}(t)$ are on the safe side, i.e. larger than some corresponding market values. However, in this article where we have pension funding in mind, such constraints are not needed.

The surplus defined in (3) will follow the dynamics given by (1) with the following specification of the contributions and initial surplus (see Steffensen (2000)),

$$
\begin{aligned}
c^{j \prime}(t) & =\left(r(t)-r^{*}(t)\right) V^{* j}(t)+\sum_{k ; k \neq j}\left(\mu^{* j k}(t)-\mu^{j k}(t)\right) R^{* j k}(t) \\
& +\sum_{k ; k \neq j} \mu^{j k}(t) R^{* j k}(t), \\
c^{j k \prime}(t) & =-R^{* j k}(t), \\
\Delta C^{j \prime}(0) & =-\Delta B^{0}(0)-V^{* 0}(0), \\
\Delta C^{j \prime}(T) & =0,
\end{aligned}
$$

where

$$
R^{* j k}(t)=b^{j k}(t)+V^{* k}(t)-V^{* j}(t) .
$$


Note that with this specification of surplus contributions we can write the dynamics of the surplus contributions for $t>0$ as

$$
\begin{aligned}
d C^{\prime}(t)= & \left(r(t)-r^{*}(t)\right) V^{* Z(t)}(t) d t+\sum_{k ; k \neq Z(t)}\left(\mu^{* Z(t) k}(t)-\mu^{Z(t) k}(t)\right) R^{* Z(t) k}(t) d t \\
& -\sum_{k \in \mathcal{I}} R^{* Z(t-) k}(t) d M^{k}(t),
\end{aligned}
$$

hereby decomposing the contribution into a systematic increment and a martingale increment. For comparison with Norberg (1999), note that he uses the letter $C$ to denote the process which contains only the systematic part of $C^{\prime}$.

We emphasize that the coefficients of the contribution process specified in (4) is just an example coming out of defining the surplus as in (3). One can easily imagine other specifications of the surplus contributions in the previous section. One obvious choice is inspired directly from the previous paragraph: Disregard the martingale term of (5) and define instead

$$
\begin{aligned}
& c^{j \prime}(t)=\left(r(t)-r^{*}(t)\right) V^{* j}(t)+\sum_{k ; k \neq j}\left(\mu^{* j k}(t)-\mu^{j k}(t)\right) R^{* j k}(t), \\
& c^{j k \prime}(t)=0 .
\end{aligned}
$$

The corresponding surplus could naturally be called the systematic surplus.

Example 1. We consider the survival model with two states corresponding to a policy holder being alive (state 0 ) or dead (state 1). For the sake of simplicity, we consider a T-year endowment insurance. In this case we can simplify the notation: $N \equiv N^{1}, \mu \equiv \mu^{01}$, and for all other quantities and functions the specification of state 0 is skipped, i.e. $b \equiv b^{0}$ (the negative premium rate), $b^{1} \equiv b^{01}$ (the life insurance sum), $\Delta B \equiv \Delta B^{0}$ (the endowment sum), $c^{\prime}(t) \equiv c^{0 \prime}(t), c^{1 \prime}(t)=c^{01^{\prime}}(t)$ etc. Assuming that we wish to control the systematic surplus, we have that

$$
\begin{aligned}
& c^{\prime}(t)=\left(\left(r-r^{*}\right) V^{*}(t)+\left(\mu^{*}(t)-\mu(t)\right)\left(b^{1}-V^{*}(\mathrm{t})\right)\right), \\
& c^{1^{\prime}}(t)=0,
\end{aligned}
$$

where

$$
V^{*}(t)=\int_{t}^{T} e^{-\int_{t}^{s} r^{*}+\mu^{*}}\left(\mu^{*}(s) b^{1}+b\right) d s+e^{-\int_{t}^{T} r^{*}+\mu^{*}} \Delta B .
$$

If, in particular the technical basis and the market basis coincide, we get that $c^{\prime}(t)=0$, and thus $C(t)=0$. 


\section{Preferences and Utility Processes}

Our scope is to search for optimal dividend payment processes. We are going to formulate our preferences over payment processes in terms of a so-called utility process $U$. Denoting by $U(t)$ the accumulated utilities over $[0, t]$, the utility process is described by

$$
d U(t)=u^{Z(t)}(t) d t+\sum_{k \in g} u^{Z(t-) k}(t) d N^{k}(t)+\sum_{n \in\{0, T\}} \Delta U^{Z(t)}(t) d \varepsilon^{n}(t) .
$$

In the utility process, $u^{j}(t)$ specifies the rate of utility in state $j$, each $u^{j k}(t)$ specifies a lump sum utility upon transitions from state $j$ to state $k$, and $\Delta U^{j}(t)$ specifies a lump sum utility during sojourns in state $j$. Below we specify how the payment process $D$ affects $U$. Our terminal goal is to find the payment process $D$ among a set of allowable payment processes that maximizes the expected total utility,

$$
E\left[\int_{0-}^{T} d U(s)\right]
$$

Note that by the introduction of the utility process $U$, we can write the expected total utility in a similar way as we usually write the expected future payments when defining the statewise reserves. In that respect we can speak of (6) as the utility reserve at time $0-$.

A utility process of the present form was introduced in Steffensen (2004). There, $u^{j}(t), u^{j k}(t)$, and $\Delta U^{j}(t)$ were defined as certain state-dependent power functions of $\delta^{\prime}(t), \delta^{k^{\prime}}(t)$, and $\Delta D^{\prime}(t)$. This set of preferences was inspired by the classical Merton problem of optimal consumption and investment. In this article the preferences are inspired by the classical pension fund optimization problem. Thus, instead we work with a quadratic dis-utility function that punishes quadratic deviations of payments from the payments in an artificial payment target process combined with quadratic deviations of surplus from zero.

For specification of the dis-utility stemming from the payment process $D$ and the surplus $X$, we introduce three further processes $A, P$, and $Q$. These processes are called the payment target process, the payment weight process, and the surplus weight process, respectively. The processes $A, P$, and $Q$ are given by

$$
\begin{aligned}
& d A(t)=a^{Z(t)}(t) d t+\sum_{k \in \mathcal{I}} a^{Z(t-) k}(t) d N^{k}(t)+\sum_{n \in\{0, T\}} \Delta A^{Z(t)}(t) d \varepsilon^{n}(t), \\
& d P(t)=p^{Z(t)}(t) d t+\sum_{k \in \mathcal{I}} p^{Z(t-) k}(t) d N^{k}(t)+\sum_{n \in\{0, T\}} \Delta P^{Z(t)}(t) d \varepsilon^{n}(t), \\
& d Q(t)=q^{Z(t)}(t) d t+\sum_{k \in \mathcal{I}} q^{Z(t-) k}(t) d N^{k}(t)+\sum_{n \in\{0, T\}} \Delta Q^{Z(t)}(t) d \varepsilon^{n}(t),
\end{aligned}
$$

and we assume that $P$ and $Q$ are increasing, i.e. all coefficients are positive. 
The preferences over the set of payments are now given by the following disutility functions

$$
\begin{aligned}
& u^{j}(t)=p^{j}(t)\left(\delta(t)-a^{j}(t)\right)^{2}+q^{j}(t) X(t)^{2}, \\
& u^{j k}(t)=p^{j k}(t)\left(\delta^{k}(t)-a^{j k}(t)\right)^{2}+q^{j k}(t) X(t)^{2}, \\
& \Delta U^{j}(t)=\Delta P^{j}(t)\left(\Delta D(t)-\Delta A^{j}(t)\right)^{2}+\Delta Q^{j}(t) X(t)^{2} .
\end{aligned}
$$

The coefficient $u^{j}(t)$ represents the rate of dis-utilities during sojourn in state $j$ at time $t$. This rate of dis-utilities stems from a deviation of $\delta(t)$ from $a^{j}(t)$ weighted with $p^{j}(t)$ and from a deviation of $X(t)$ from 0 weighted with $q^{j}(t)$. The coefficient $u^{j k}(t)$ represents the lump sum dis-utility when jumping from state $j$ to state $k$ at time $t$. This lump sum dis-utility stems from a deviation of $\delta^{k}(t)$ from $a^{j k}(t)$ weighted with $p^{j k}(t)$ and from a deviation of $X(t)$ from 0 weighted with $q^{j k}(t)$. Finally, the coefficient $\Delta U^{j}(t)$ represents a lump sum dis-utility at a deterministic point in time during sojourn in state $j$. This lump sum dis-utility stems from a deviation of $\Delta D(t)$ from $\Delta A^{j}(t)$ weighted with $\Delta P^{j}(t)$ and from a deviation of $X(t)$ from 0 weighted with $\Delta Q^{j}(t)$. We allow for lump sum dis-utility at the deterministic time points 0 and $T$ only. One may find it odd to add lump sum dis-utility corresponding to $q^{j k}$ and $\Delta Q^{j}$. Actually, these are also a burden from a mathematical point of view as we see below. However, for the sake of symmetry, we keep them as far as we can.

Note that the payment processes $A, P$, and $Q$ are not in general real payment processes experienced by the policy holder or the insurance company. Their only role is to specify the preferences over payment streams $D$. Thus, we could simply have introduced all the coefficients of $A, P$, and $Q$ directly as state-dependent functions. However, for the comprehension of the structure of these coefficients it is beneficial to have these artificial payment processes in mind. Furthermore, whereas $P$ and $Q$ really have not much to do with payments, the process $A$ may be equal or related to a real payment process.

One may suggest the introduction of a surplus target process $Y$, say, with dynamics given by

$$
d Y(t)=y^{Z(t)}(t) d t+\sum_{k \in \mathcal{I}} y^{Z(t-) k}(t) d N^{k}(t)+\sum_{n \in\{0, T\}} \Delta Y^{Z(t)}(t) d \varepsilon^{n}(t)
$$

and replace $X(t)^{2}$ by $(X(t)-Y(t))^{2}$ in the coefficients of the utility process. This formulation, however, is covered by the construction above by simply redefining the surplus and the contribution processes by

$$
\begin{aligned}
\tilde{X} & =X-Y, \\
\tilde{C} & =C-Y .
\end{aligned}
$$

We end this section with a comment on the idea of penalizing deviations of $X$ from 0 . At the end of the previous section we emphasized that one could choose 
to work with several notions of surplus. Obviously, controlling different notions of surplus would have to be motivated in different ways. Consider the individual surplus introduced in the previous section, containing both a systematic and an erratic term. A policy holder contributes to this surplus in two different ways. Firstly, he contributes to the surplus systematically according to the difference between the technical assumptions and realized mortality and return. Secondly, he contributes erratically according to his own course of life. If we control the individual surplus, we think that his dividends should be affected, not only by the systematic contributions, but also by this course of life. Controlling the individual surplus reduces the risk of the other parties involved i.e. the insurance company and/or the employers, compared to the alternative below.

Alternatively, consider the systematic surplus explained at the end of the previous section. This surplus simply disregards the erratic term of the individual surplus. Now, the policy holder does not contribute to the surplus erratically by his course of life. Only systematic contributions are accounted for and, thus, distributed in terms of dividends. This construction leaves all the unsystematic risk to the other parties involved.

One cannot say that one construction is right and the other is wrong. They are just different constructions based on different ideas with different levels of insurance in the sense of averaging away the risk of the policy holder. As the preferences over the surplus should be interpreted differently for different notions of surplus, so should also possible constraints on $X$. We return to constraints on $X$ in Section 7.

\section{Markov Chain Pension Fund Optimization}

We define the optimal value function $V$ by

$$
V^{j}(t, x)=\inf _{D \in \mathcal{A}} E_{t, x, j}\left[\int_{t}^{T} d U(s)\right],
$$

where $E_{t, x, j}$ denotes conditional expectation given that $X(t)=x$ and $Z(t)=j$. We can speak of $V^{j}(t, x)$ as the statewise optimal value function.

A fundamental system of differential equations in control theory is the Bellman system for the optimal value function. The Bellman system is here given as the infimum over admissible controls of partial differential equations for the optimal value function. We shall not derive the Bellman equation here but refer to Steffensen (2000) for a derivation of partial differential equations for relevant conditional expected values. It can be realized that for all $j \in \mathcal{I}$,

$$
\begin{aligned}
V_{t}^{j}(t, x)=\sup _{\delta, \delta^{k}, k \neq j} & {\left[-V_{x}^{j}(t, x)\left(c^{j}(t)-\delta\right)-p^{j}(t)\left(\delta-a^{j}(t)\right)^{2}-q^{j}(t) x^{2}\right.} \\
& \left.-\sum_{k ; k \neq j} \mu^{j k}(t) R^{j k}(t, x)\right]
\end{aligned}
$$


and for $t \in\{0, T\}$,

$$
0=\sup _{\Delta D}\left[-\Delta R^{j}(t, x)\right]
$$

where subscript denotes the partial derivative and where

$$
\begin{aligned}
R^{j k}(t, x)= & p^{j k}(t)\left(\delta^{k}-a^{j k}(t)\right)^{2} \\
& +q^{j k}(t)\left(x+c^{j k}(t)-\delta^{k}\right)^{2} \\
& +V^{k}\left(t, x+c^{j k}(t)-\delta^{k}\right)-V^{j}(t, x) \\
\Delta R^{j}(t, x)= & \Delta P^{j}(t)\left(\Delta D-\Delta A^{j}(t)\right)^{2} \\
& +\Delta Q^{j}(t)\left(x+\Delta C^{j}(t)-\Delta D\right)^{2} \\
& +V^{j}\left(t, x+\Delta C^{j}(t)-\Delta D\right)-V^{j}(t-, x) .
\end{aligned}
$$

The differential equality in (8) maximizes the partial derivative in $t$ at any point in the state space and the equality in (9) maximizes the jump in the value function at time 0 and at time $T$. Together the equalities minimize the conditional expected value in (7) and hence characterize the value function.

It should be emphasized that the Bellman system is actually a system of $J$ differential equations with $J$ conditions at time 0 and at time $T$. The Bellman system contains the terms present in the Bellman equation for the classical pension fund optimization problem and an additional term stemming from the uncertainty in the process $Z$.

The system of $J$ differential equations is comparable with the classical socalled Thiele's differential equation for the state wise reserves, see e.g. Steffensen (2000). This motivates partly the notation $V^{j}$ and $R^{j k}$ : The statewise reserve is usually denoted by $V^{j}$ and the risk sum in Thiele's differential equation is usually denoted by $R^{j k}$. Here, the contents of these terms is different but the structure is partly the same. The term $\Delta R^{j}(t, x)$ has similarities with a risk sum and is used to specify the development of the utility reserve at deterministic points in time with a lump sum dis-utility. For a given lump sum $\Delta D$, the relation $\Delta R^{j}(t, x)=0$ updates the utility reserve at such a point in time. E.g. at time $T$, since $V^{j}(T, x)=0$ for all $x$, the relation gives the terminal condition

$$
V^{j}(T-, x)=\Delta P^{j}(T)\left(\Delta D-\Delta A^{j}(T)\right)^{2}+\Delta Q^{j}(T)\left(x+\Delta C^{j}(T)-\Delta D\right)^{2} .
$$

The Bellman equation plays two different roles in control theory. One role is that if the optimal value function is sufficiently smooth, then this function satisfies the Bellman system. However, usually it is very difficult to prove a priori the smoothness conditions. Instead one often works with the verification result stating that a sufficiently nice function solving the Bellman system actually coincides with the optimal value function. In fact, it is not even necessary to come up with a classical solution to the Bellman system. One just needs a so-called viscosity solution with relaxed requirements on differentiability which will then coincide with the optimal value function. 
We now guess a solution to the Bellman system based on a separation of $x$ in the same way as in the classical case. We try the solution

$$
V^{j}(t, x)=f^{j}(t)\left(x-g^{j}(t)\right)^{2}+h^{j}(t) .
$$

This form leads to the following list of partial derivatives,

$$
\begin{aligned}
V_{t}^{j}(t, x) & =f_{t}^{j}(t)\left(x-g^{j}(t)\right)^{2}-2 f^{j}(t) g_{t}^{j}(t)\left(x-g^{j}(t)\right)+h_{t}^{j}(t), \\
V_{x}^{j}(t, x) & =2 f^{j}(t)\left(x-g^{j}(t)\right) .
\end{aligned}
$$

A candidate for the optimal $D$ is found by solving (8) for the suprema with respect to the decision variables in $D$, for $j, k \in \mathcal{J}, k \neq j$,

$$
\begin{aligned}
0= & 2 f^{j}(t)\left(x-g^{j}(t)\right)-2 p^{j}(t)\left(\delta-a^{j}(t)\right), \\
0= & 2 p^{j k}(t)\left(\delta^{k}-a^{j k}(t)\right)-2 q^{j k}(t)\left(x+c^{j k}(t)-\delta^{k}\right) \\
& -2 f^{k}(t)\left(x+c^{j k}(t)-\delta^{k}-g^{k}(t)\right), \\
0= & 2 \Delta P^{j}(t)\left(\Delta D-\Delta A^{j}(t)\right)-2 \Delta Q^{j}(t)\left(x+\Delta C^{j}(t)-\Delta D\right) \\
& -2 f^{j}(t)\left(x+\Delta C^{j}(t)-\Delta D-g^{j}(t)\right) .
\end{aligned}
$$

This leads to the candidates, abbreviating $S^{j k}(t)=p^{j k}(t)+q^{j k}(t)+f^{k}(t)$ and $\Delta S^{j}(t)$ $=\Delta P^{j}(t)+\Delta Q^{j}(t)+f^{j}(t)$,

$$
\begin{aligned}
\delta^{j}(t, x) & =a^{j}(t)+\frac{f^{j}(t)}{p^{j}(t)}\left(x-g^{j}(t)\right), \\
\delta^{j k}(t, x) & =\frac{p^{j k}(t)}{S^{j k}(t)} a^{j k}(t)+\frac{q^{j k}(t)}{S^{j k}(t)}\left(x+c^{j k}(t)\right)+\frac{f^{k}(t)}{S^{j k}(t)}\left(x+c^{j k}(t)-g^{k}(t)\right), \\
\Delta D^{j}(t, x) & =\frac{\Delta P^{j}(t)}{\Delta S^{j}(t)} \Delta A^{j}(t)+\frac{\Delta Q^{j}(t)}{\Delta S^{j}(t)}\left(x+\Delta C^{j}(t)\right)+\frac{f^{j}(t)}{\Delta S^{j}(t)}\left(x+\Delta C^{j}(t)-g^{j}(t)\right),
\end{aligned}
$$

where the notation is evident and exposes $\delta, \delta^{k}, \Delta D$ as functions of $(t, j, x)$.

The optimal control variables in (10) can be interpreted as follows:

$\delta^{j}(t)$ is equal to its target $a^{j}(t)$ adjusted with a correction term which takes into account the future. $X(t)$ is corrected towards $g^{j}(t)$, and the ratio $\frac{f^{j}(t)}{p^{j}(t)}$ determines the weight of this correction. If $p^{j}(t)$ is large (relative to $f^{j}(t)$ ), there is a high consideration for the present preference to have $\delta^{j}$ close to $a^{j}$, and vice versa. $\delta^{j k}(t)$ is a weighted average of three considerations. Firstly, $\delta^{j k}(t)$ is preferred to be close to $a^{j k}(t)$ and this is weighted with $p^{j k}(t)$. Secondly, after a possible jump from $j$ to $k, X(t-)+c^{j k}(t)$ (the position after the jump but before controlling) is preferred to be close to 0 and this is weighted with $q^{j k}(t)$. Thirdly, also the future after the jump must be taken into consideration and for this 
$X(t-)+c^{j k}(t)$ should be corrected towards $g^{k}(t)$, and $f^{k}(t)$ determines the weight of this correction.

$\Delta D^{j}(t)$ is a weighted average somewhat similar to $\delta^{j k}(t)$. Firstly, $\Delta D^{j}(t)$ is preferred to be close to $\Delta A^{j}(t)$ and this is weighted with $\Delta P^{j}(t)$. Secondly, at time $t, X(t-)+\Delta C^{j}(t)$ (the position at time $t$ but before controlling) is preferred to be close to 0 and this is weighted with $\Delta Q^{j}(t)$. Thirdly, also the future must be taken into consideration and for this $X(t-)+\Delta C^{j}(t)$ should be corrected towards $g^{j}(t)$, and $f^{j}(t)$ determines the weight of this correction.

We see that both $\delta^{j}, \delta^{j k}$, and $\Delta D^{j}$ are linear functions of the surplus as the controllable parameter is it in the classical case. However, the coefficients involve the payment processes $A$ and $C$ and the functions $f$ and $g$. Inserting the optimal candidate in the Bellman system gives, after several rearrangements, the following partial differential equations for $f^{j}(t)$ and $g^{j}(t)$,

$$
\begin{aligned}
f_{t}^{j}(t) & =\frac{f^{j}(t)^{2}}{p^{j}(t)}-q^{j}(t)-\sum_{k ; k \neq j} \mu^{j k}(t) R^{f ; j k}(t), \\
0 & =\Delta R^{f ; j}(t), t \in\{0, T\}, \\
g_{t}^{j}(t) & =r^{* j}(t) g^{j}(t)+c^{j}(t)-a^{j}(t)-\sum_{k ; k \neq j} \mu^{j k}(t) R^{g ; j k}(t), \\
0 & =\Delta R^{g ; j}(t), t \in\{0, T\},
\end{aligned}
$$

where

$$
r^{* j}(t)=\frac{q^{j}(t)}{f^{j}(t)},
$$

and the risk sums in the differential equations for $f$ and $g$ are given by

$$
\begin{gathered}
R^{f ; j k}(t)=\frac{p^{j k}(t)}{S^{j k}(t)}\left(q^{j k}(t)+f^{k}(t)\right)-f^{j}(t), \\
\Delta R^{f ; j}(t)=\frac{\Delta P^{j}(t)}{\Delta S^{j}(t)}\left(\Delta Q^{j}(t)+f^{j}(t)\right)-f^{j}(t-), \\
R^{g ; j k}(t)=\frac{p^{j k}(t)}{S^{j k}(t)}\left(\frac{q^{j k}(t)+f^{k}(t)}{f^{j}(t)}\left(a^{j k}(t)-c^{j k}(t)-g^{j}(t)\right)+\frac{f^{k}(t)}{f^{j}(t)} g^{k}(t)\right) \\
=\frac{R^{f ; j k}(t)+f^{j}(t)}{f^{j}(t)}\left(a^{j k}(t)-c^{j k}(t)-g^{j}(t)\right) \\
+\frac{R^{f ; j k}(t)+f^{j}(t)}{q^{j k}(t)+f^{k}(t)} \frac{f^{k}(t)}{f^{j}(t)} g^{k}(t),
\end{gathered}
$$




$$
\begin{aligned}
\Delta R^{g ; j}(t)= & \frac{\Delta P^{j}(t)}{\Delta S^{j}(t)}\left(\frac{\Delta Q^{j}(t)+f^{j}(t)}{f^{j}(t-)}\left(\Delta A^{j}(t)-\Delta C^{j}(t)-g^{j}(t-)\right)+\frac{f^{j}(t)}{f^{j}(t-)} g^{j}(t)\right) \\
= & \frac{\Delta R^{f ; j}(t)+f^{j}(t-)}{f^{j}(t-)}\left(\Delta A^{j}(t)-\Delta C^{j}(t)-g^{j}(t-)\right) \\
& +\frac{\Delta R^{f ; j}(t)+f^{j}(t-)}{\Delta Q^{j}(t)+f^{j}(t)} \frac{f^{j}(t)}{f^{j}(t-)} g^{j}(t) \\
= & \Delta A^{j}(t)-\Delta C^{j}(t)-g^{j}(t-)+\frac{f^{j}(t)}{\Delta Q^{j}(t)+f^{j}(t)} g^{j}(t) .
\end{aligned}
$$

The last equality follows from (11). The system of differential equations for $f$ is a $J$-dimensional Riccati equation. For a given terminal condition, this has a unique positive solution under certain assumptions on the coefficients. We see that the terminal condition for $f$ must be $f^{j}(T)=0$, whereas we take the terminal condition for $g$ to be $g^{j}(T)=0$ by convention. We emphasize that we could introduce any terminal condition for $g$. Following (11), (12), (13), and (14), the terminal conditions $f^{j}(T)=0$ and $g^{j}(T)=0$ lead to

$$
\begin{aligned}
& f^{j}(T-)=\frac{\Delta P^{j}(T) \Delta Q^{j}(T)}{\Delta P^{j}(T)+\Delta Q^{j}(T)}, \\
& g^{j}(T-)=\Delta A^{j}(T)-\Delta C^{j}(T) .
\end{aligned}
$$

Given $f$, the system of differential equations for $g$ has similarities with Thiele's differential equation, see Steffensen (2000). However, the quantity $R^{g ; j k}$ is not a risk sum in the same sense as in Thiele's differential equation and $\Delta R^{g ; j}(t)$ does not lead to a usual adjustment of the conditional expected value for a lump sum payment at a deterministic point in time. Nevertheless, it is possible to derive a stochastic representation formula for the solution to the differential equation in the case where $q^{j k}(t)=\Delta Q^{j}(t)=0$. This is done in the rest of this section.

In the case $q^{j k}(t)=\Delta Q^{j}(t)=0, R^{f ; j k}(t), \Delta R^{f ; j}(t), R^{g ; j k}(t)$ and $\Delta R^{g ; j k}(t)$ above simplify to

$$
\begin{aligned}
R^{f ; j k}(t) & =f^{k}(t)-f^{j}(t), \\
\Delta R^{f ; j}(t) & =f^{j}(t)-f^{j}(t-), \\
R^{g ; j k}(t) & =\frac{p^{j k}(t)}{p^{j k}(t)+f^{k}(t)} \frac{f^{k}(t)}{f^{j}(t)}\left(a^{j k}(t)-c^{j k}(t)-g^{j}(t)+g^{k}(t)\right), \\
\Delta R^{g ; j}(t) & =\Delta A^{j}(t)-\Delta C^{j}(t)-g^{j}(t-)+g^{j}(t) .
\end{aligned}
$$

One realizes then that $g$ can be written as a conditional expectation of the present value of the payment process $A$ - $C$ under the particular state-dependent discount rate $r^{*}$ and under a particular measure $P^{*}$ defined below, i.e. 


$$
g^{j}(t)=E_{t, j}^{*}\left[\int_{t}^{T} e^{-\int_{t}^{s} r^{* Z(u)}(u) d u} d(A-C)(s)\right],
$$

where $E^{*}$ denotes expectation with respect to the measure $P^{*}$.

Define the likelihood process $L$ and the corresponding jump kernel by

$$
\begin{aligned}
& d L(t)=L(t-) \sum_{k \in \mathcal{J}} \gamma^{Z(t-) k}(t)\left(d N^{k}(t)-\mu^{Z(t) k}(t) d t\right), \\
& \gamma^{j k}(t)=\frac{p^{j k}(t)}{p^{j k}(t)+f^{k}(t)} \frac{f^{k}(t)}{f^{j}(t)}-1, j \neq k .
\end{aligned}
$$

Then we can change measure from $P$ to $P^{*}$ by the definition $L_{T}=\frac{d P^{*}}{d P}$, and it follows from Girsanov's theorems (see e.g. Björk (2004)) that $N^{k}$ under $P^{*}$ admits the intensity process

$$
\mu^{* Z(t-) k}(t)=\left(1+\gamma^{Z(t-) k}(t)\right) \mu^{Z(t-) k}(t) .
$$

We can finally write

$$
\begin{aligned}
g_{t}^{j}(t) & =r^{* j}(t) g^{j}(t)+c^{j}(t)-a^{j}(t)-\sum_{k ; k \neq j} \mu^{* j k}(t) R^{g ; * j k}(t), \\
g^{j}(T-) & =\Delta A^{j}(T)-\Delta C^{j}(T), \\
R^{g ; * j k}(t) & =a^{j k}(t)-c^{j k}(t)+g^{k}(t)-g^{j}(t),
\end{aligned}
$$

which is precisely a version of Thiele's differential equation for a reserve defined by (15).

Example 2. We now continue Example 1. We are now interested in paying out dividends optimally and need for this purpose to specify the three processes $A, P$, and $Q$. We take the target process $A$ to be 0 . This means that the policy holder has a target process for his total payments $B+D$ equal to $B$. For the weight process $P$, we take the coefficients corresponding to state 1 (dead) to be positive. We skip the specification of state 0 for the other elements of $P$ and take $p \equiv p^{0}$, $p^{1} \equiv p^{01}$, and $\Delta P \equiv \Delta P^{0}$ to be constant. For the weight process $Q$ we take the coefficients corresponding to state 1 to be zero. Furthermore, we take $q \equiv q^{0}, q^{1} \equiv q^{01}$, and $\Delta Q \equiv \Delta Q^{0}$ to be constant. Plugging in all these coefficients in the differential equations for $f$ and $g$, we see that the differential equations corresponding to state 1 are solved by $f^{1}=g^{1}=0$. Hereafter, the differential equations and terminal conditions for $f \equiv f^{0}$ and $g \equiv g^{0}$ are reduced to 


$$
\begin{aligned}
f_{t}(t) & =\frac{f(t)^{2}}{p}-\mu(t)\left(\frac{p^{1} q^{1}}{p^{1}+q^{1}}-f(t)\right)-q, \\
f(T-) & =\frac{\Delta P \Delta Q}{\Delta P+\Delta Q}, \\
g_{t}(t) & =\left(\frac{q}{f(t)}+\mu^{*}(t)\right) g(t)+c(t), \\
g(T) & =0
\end{aligned}
$$

The Riccati equation for $f$ must be solved numerically, while the solution for $g$ is given by

$$
g(t)=-\int_{t}^{T} e^{-\int_{t}^{s} \frac{q}{f}+\mu} c(s) d s
$$

We can now write down the optimal dividend payments in terms of $f$ and $g$,

$$
\begin{aligned}
\delta(t, x) & =\frac{f(t)}{p}(x-g(t)), \\
\delta^{1}(x) & =\frac{q^{1}}{p^{1}+q^{1}} x \\
\Delta D^{1}(x) & =\frac{\Delta Q}{\Delta P+\Delta Q} x .
\end{aligned}
$$

The lump sum dividend payments upon death or termination whatever occurs first, have the same structure. It is easy to verify that these dividend payments simply minimize the final lump sum penalties given by $p^{1}\left(\delta^{1}\right)^{2}+q^{1}\left(x-\delta^{1}\right)^{2}$ and $\Delta P \Delta D^{2}+\Delta Q(x-\Delta D)^{2}$, respectively. The ratio $q^{1} /\left(p^{1}+q^{1}\right)$ determines the preferences between two extreme situations: Either one could get $\delta^{1}=0$ by having no preferences for $X$ i.e. $q^{1}=0$, or one could obtain $X=0$ after death or termination whatever occurs first, by having no preferences for $\delta^{1}$, i.e. $p^{1}=0 . A$ similar interpretation goes for the ratio $\Delta Q /(\Delta P+\Delta Q)$.

\section{Constrained Payments}

In section 5 we had no constraints on the dividend payments. In this section we show how it is possible to solve problems where certain payments are constrained to be equal to certain values. One can think of several examples where such constraints are relevant. Consider the optimization problem under the constraint that for $s \leq t \leq u$

$$
\delta(t)=\hat{a}^{Z(t)}(t)
$$


Under this constraint, we have that the term in $d U(t)$ involving $\delta(t)$ for $s \leq t \leq u$ is given by

$$
p^{Z(t)}(t)\left(\hat{a}^{Z(t)}(t)-a^{Z(t)}(t)\right)^{2} d t .
$$

For a given weight $p^{Z(t)}(t)$ and a given target $a^{Z(t)}(t)$, this is a deterministic function of $(t, Z(t))$ and therefore plays no role for the decision of the optimal strategy. We can therefore choose $p^{Z(t)}(t)$ and $a^{Z(t)}(t)$ freely, and in particular search for coefficients such that the unconstrained problem has a solution where the constraint is fulfilled for the optimal dividend process. If we find such, we have then a solution for our constrained problem. From the optimal dividends in the unconstrained problem (10) we see that if we for $s \leq t \leq u$ choose

$$
\begin{aligned}
& p^{Z(t)}(t)=v, \\
& a^{Z(t)}(t)=\hat{a}^{Z(t)}(t),
\end{aligned}
$$

and let $v \rightarrow \infty$, then we get in the limit that for $s \leq t \leq u$ (and all other weights and targets fixed),

$$
\frac{f^{Z(t)}(t)}{p^{Z(t)}(t)}=0
$$

and, thus,

$$
\delta(t)=\hat{a}^{Z(t)}(t)
$$

such that the constraint is fulfilled. The artificial set of weight and target functions given in (16) has the obvious interpretation that, in the limit, deviations from the target given by the constraint are punished infinitesimally severely. Obviously, to avoid an infinite value function in the limit, the constraint is therefore fulfilled by the optimal control.

For constraints on lump sum payments upon transition and at deterministic points in time the argument goes in almost the same way. Here we go through the argument for a constrained lump sum payment upon transition. Consider the optimization problem under the constraint that

$$
\delta^{k}(t)=\hat{a}^{Z(t-) k}(t)
$$

Under this constraint, we have that the term in $d U(t)$ involving $\delta^{k}(t)$ is given by

$$
\sum_{k \in \mathcal{J}} p^{Z(t-) k}(t)\left(\hat{a}^{Z(t-) k}(t)-a^{Z(t-) k}(t)\right)^{2} d N^{k}(t) .
$$

For a given weight $p^{Z(t-) k}(t)$ and a given target $a^{Z(t-) k}(t)$, this is a deterministic function of $(t, Z(t-))$ and therefore plays no role for the decision of the optimal 
strategy. We can therefore choose $p^{Z(t-) k}(t)$ and $a^{Z(t-) k}(t)$ freely. If we find coefficients such that the unconstrained problem has a solution where the constraint is fulfilled for the optimal dividend proces, then we have a solution for our constrained problem. From the optimal dividends in the unconstrained problem (10) we see that if we choose

$$
\begin{aligned}
& p^{Z(t-) k}(t)=v, \\
& a^{Z(t-) k}(t)=\hat{a}^{Z(t-) k}(t),
\end{aligned}
$$

and let $v \rightarrow \infty$, then we get in the limit that (for all other weights and targets fixed)

$$
\frac{p^{j k}(t)}{S^{j k}(t)}=1, \frac{q^{j k}(t)}{S^{j k}(t)}=0, \frac{f^{k}(t)}{S^{j k}(t)}=0
$$

and, thus,

$$
\delta^{k}(t)=\hat{a}^{Z(t-) k}(t)
$$

such that the constraint is fulfilled. Again, the interpretation of the weight and target functions given in (17) is that deviations from the target are punished infinitesimally severely in the limit. To avoid an infinite value function, the constraint is therefore fulfilled by the optimal control.

Example 3. We now continue Example 2. Consider the case where the dividend rate is constrained to be zero. This case could appropriately be spoken of as Defined Contribution since the premium rate is not regulated through dividends but benefits are. This is handled by considering the controls for $p \rightarrow \infty$. The optimal dividends in the limit turn into

$$
\begin{aligned}
\delta(t, x) & =0, \\
\delta^{1}(t, x) & =\frac{q^{1}}{p^{1}+q^{1}} x, \\
\Delta D^{1}(T, x) & =\frac{\Delta Q}{\Delta P+\Delta Q} x .
\end{aligned}
$$

Alternatively, consider the case where lump sum dividends are constraint to be zero. This case could appropriately be spoken of as Defined Benefits since the benefits are not regulated through dividends but the premium rate is. This is handled by considering the controls for $p^{1} \rightarrow \infty$ and $\Delta P \rightarrow \infty$. Then the optimal dividends in the limit turn into

$$
\begin{aligned}
\delta(t, x) & =\frac{f(t)}{p}(x-g(t)), \\
\delta^{1}(t, x) & =0, \\
\Delta D^{1}(T, x) & =0 .
\end{aligned}
$$


The differential equation for $f$ becomes

$$
\begin{aligned}
f_{t}(t) & =\frac{f(t)^{2}}{p}-\mu(t)\left(q^{1}-f(t)\right)-q, \\
f(T-) & =\Delta Q .
\end{aligned}
$$

\section{Constrained Surplus}

In this section we explain how it is possible to solve problems where the terminal surplus is constrained to equal zero. If $X$ is the systematic surplus, this relates to the individual fairness criterion as described by Norberg (1999) since this constraint sees to it that the surplus is emptied completely at termination for a given insurance contract or portfolio of contracts. Thus, we consider the optimization problem under the constraint that

$$
X(T)=0 .
$$

Under this constraint, we have that the term in $d U(T)$ involving $X(T)$ equals 0 . Therefore, for a given weight $\Delta Q^{j}(T)$, this plays no role for the decision of the optimal strategy. We can therefore choose $\Delta Q^{j}(T)$ freely. If we find a coefficients such that the unconstrained problem has a solution where the constraint is fulfilled for the optimal dividend process, then we have a solution for our constrained problem. From the optimal dividends in the unconstrained problem (10) we see that if we choose

$$
\Delta Q^{j}(T)=v,
$$

and let $v \rightarrow \infty$, then we get in the limit that (for all other weights and targets fixed)

$$
\frac{\Delta P^{j}(T)}{\Delta S^{j}(T)}=0, \frac{\Delta Q^{j}(T)}{\Delta S^{j}(T)}=1, \frac{f^{j}(T)}{\Delta S^{j}(T)}=0,
$$

and, thus,

$$
\Delta D^{j}(T)=X(T-)+\Delta C^{j}(T),
$$

leading to

$$
X(T)=X(T-)+\Delta C^{Z(T)}(T)-\Delta D^{Z(T)}(T)=0 .
$$

which exactly obeys our constraint. The artificial weight given in (18) has the obvious interpretation that in the limit, deviation from zero is punished infinitesimally severely. Obviously, to avoid an infinite value function in the limit, the constraint is then fulfilled. 
Maybe even more interesting is a situation where the constraint $X(T)=0$ is combined with a constraint on the terminal lump sum dividend

$$
\Delta D(T)=\Delta \hat{A}^{Z(T)}(T) .
$$

Then

$$
d U(T)=\Delta P^{Z(T)}(T)\left(\Delta \hat{A}^{Z(T)}(T)-\Delta A^{Z(T)}(T)\right)^{2},
$$

and the ideas in the present and the previous sections are combined by choosing

$$
\begin{aligned}
\Delta P^{j}(T) & =v, \\
\Delta A^{Z(t)}(t) & =\Delta \hat{A}^{Z(T)}(T), \\
\Delta Q^{j}(T) & =v,
\end{aligned}
$$

and examine what happens for $v \rightarrow \infty$. Consider the terminal dividend payment $\Delta D^{j}(T)$ and the terminal value of $X(T)$. Since $f^{j}(T)=0$ and $\Delta P^{j}(T) / \Delta S^{j}(T)=$ $\Delta Q^{j}(T) / \Delta S^{j}(T)=1 / 2$ for all $v$, we get

$$
\begin{aligned}
\Delta D^{j}(T) & =\frac{1}{2} \Delta \hat{A}^{j}(T)+\frac{1}{2}\left(X(T-)+\Delta C^{j}(T)\right), \\
X(T) & =X(T-)+\Delta C^{Z(T)}(T)-\Delta D^{Z(T)}(T) .
\end{aligned}
$$

Putting these relations together we conclude that if $X(T-)=\Delta \hat{A}^{Z(T)}(T)-\Delta C^{Z(T)}(T)$, then

$$
\begin{aligned}
\Delta D^{j}(T) & =\Delta \hat{A}^{j}(T), \\
X(T) & =0,
\end{aligned}
$$

which exactly obeys our constraint. Thus, it remains to argue that $X(T-)=$ $\Delta \hat{A}^{Z(T)}(T)-\Delta C^{Z(T)}(T)$. However, since for $v \rightarrow \infty$,

$$
f^{j}(T-)=\frac{\Delta P^{j}(T) \Delta Q^{j}(T)}{\Delta P^{j}(T)+\Delta Q^{j}(T)}=\frac{v}{2} \rightarrow \infty,
$$

we see that the continuous dividend rate $\frac{f^{j}(t)}{p^{j}(t)}\left(x-g^{j}(t)\right)$ pushes $X$ towards $g$ infinitesimally fast as $t \rightarrow T$ (for all other weights and targets fixed). Since $g^{j}(T-)=$ $\Delta \hat{A}^{Z(T)}(T)-\Delta C^{j}(T)$, we have indeed $X(T-)=\Delta \hat{A}^{Z(T)}(T)-\Delta C^{Z(T)}(T)$ in the limit, and we are done.

An alternative to the constraint $X(T)=0$ is to constrain the expected value of $X(T)$, e.g. $E[X(T)]=0$. If $X$ is the systematic surplus, this relates to the mean portfolio fairness criterion as described by Norberg (1999) since this constraint sees to it that the surplus is emptied in expectation at termination 
for a given insurance contract or portfolio of contracts. We just mention here that this type of constraint can be approached by a so-called Lagrange multiplier. Steffensen (2001) contains results for the classical optimal pension funding set-up in this direction. The techniques used there also applies to the control problem studied in this article.

Example 4. We now return to Example 2. Consider the case where the surplus at time $T$ is constrained to be zero. This is handled by considering the controls for $q^{1} \rightarrow \infty$ and $\Delta Q \rightarrow \infty$. Then the optimal dividends in the limit turn into

$$
\begin{aligned}
\delta(t, x) & =\frac{f(t)}{p}(x-g(t)), \\
\delta^{1}(t, x) & =x, \\
\Delta D^{1}(T, x) & =x .
\end{aligned}
$$

The differential equation for $f$ becomes

$$
\begin{aligned}
f_{t}(t) & =\frac{f(t)^{2}}{p}-\mu(t)\left(p^{1}-f(t)\right)-q, \\
f(T-) & =\Delta P .
\end{aligned}
$$

Note, in particular, that upon death or termination whichever occurs first, the surplus $X$ is fully paid out as lump sum dividend in order to fulfill the constraint $X(T)=0$.

\section{ACKNOWLEDGMENTS}

The author wishes to thank two anonymous referees for several useful comments.

\section{REFERENCES}

BJöRK, T. (2004) Arbitrage Theory in Continuous Time. Oxford University Press.

CAIRns, A.J.G. (2000) Some notes on the dynamics and optimal control of stochastic pension fund models in continuous time. ASTIN Bulletin, 30(1), 19-55.

DuFRESNE, D. (1989) Stability of pension systems when rates of return are random. Insurance: Mathematics and Economics, 8, 71-76.

Haberman, S. and Sung, J.-H. (1994) Dynamic approaches to pension funding. Insurance: Mathematics and Ecnonomics, 15, 151-162.

Hoem, J.M. (1969) Markov chain models in life insurance. Blätter der Deutschen Gesellschaft für Versicherungsmathematik, 9, 91-107.

Merton, R.C. (1969) Lifetime portfolio selection under uncertainty: The continuous time case. Review of Economics and Statistics, 51, 247-257.

Norberg, R. (1999) A theory of bonus in life insurance. Finance and Stochastics, 3(4), 373-390.

O'Brien, T. (1986) A stochastic-dynamic approach to pension funding. Insurance: Mathematics and Economics, 5, 141-146.

Steffensen, M. (2000) Contingent claims analysis in life and pension insurance. Proceedings AFIR 2000, pages 587-603. 
Steffensen, M. (2001) On valuation and control in life and pension insurance. Ph.D.-thesis, Laboratory of Actuarial Mathematics, University of Copenhagen.

Steffensen, M. (2004) On Merton's problem for life insurers. ASTIN Bulletin, 34(1).

\section{Mogens STEFFEnSEN}

Laboratory of Actuarial Mathematics

Institute of Mathematical Sciences

University of Copenhagen

Universitetsparken 5

DK-2100 Copenhagen Ø, Denmark

E-mail:mogens@math.ku.dk 